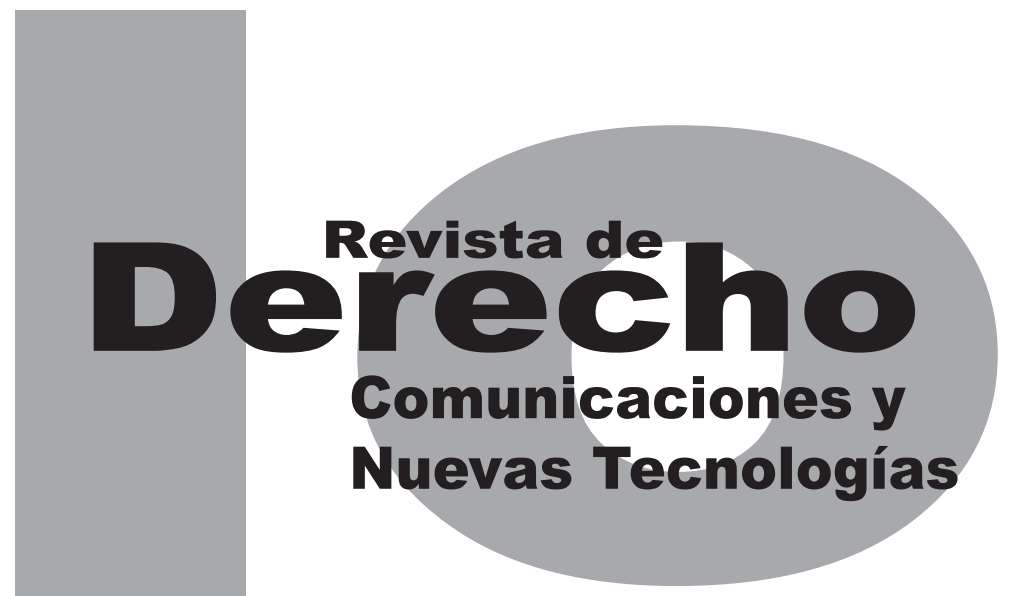

\title{
INTERNET COMO MEDIO DE PROMOCIÓN DE LA DEMOCRACIA
}

\author{
TAtiana MonSALVe López
}

Artículo de reflexión

DOI: http://dx.doi.org/10.15425/redecom.13.2015.04

Universidad de los Andes

Facultad de Derecho

Revista de Derecho, Comunicaciones y Nuevas Tecnologías

No.13, Enero - Junio de 2015. ISSN 1909-7786 


\title{
Internet como medio de promoción de la democracia
}

\section{Resumen}

Internet es una tic que ha impactado el mundo en prácticamente todos los escenarios (social, político, económico, cultural, etc.). En el presente escrito se pretende demostrar que Internet sí puede incidir de manera positiva en la democracia. Para ello, se realizará, en primer lugar, un estado del arte histórico de la intervención de la tecnología en la política, y posteriormente se expondrán las bases estructurales de la democracia electrónica para, finalmente, analizar las debilidades de la figura. Lo anterior, con el fin de evidenciar que gracias a la tecnología, la participación ciudadana se ve facilitada e incentivada.

Palabras clave: Internet, democracia electrónica, sociedad de la información, participación política, tecnologías de la información y las comunicaciones (TIC), democracia directa, democracia representativa, participación ciudadana.

\section{Internet, a tool to promote Democracy}

\begin{abstract}
Internet has an impact in almost every aspect of the society worldwide. This paper intends to demonstrate that this ICT can have a positive influence to strengthen democracies. It presents a brief historical resume of technology's intervention in politics. Subsequently, it explains the structural basis of electronic democracy. Finally, it analyses the challenges that technology must address in order to facilitate democratic participation
\end{abstract}

Keywords: Internet, e-democracy, society of information, information technology of information and communication (ICT), direct democracy, representative democracy, citizen participation.

\section{A internet como meio de promoção da democracia}

\section{Resumo}

A internet é uma tıc que tem impactado o mundo em praticamente todos os cenários (social, político, econômico, cultural, etc.). No presente escrito se pretende demostrar que a internet pode incidir de maneira positiva na democracia. Para isso, será realizado, em primeiro lugar, um estado da arte histórica da intervenção da tecnologia na política, e posteriormente serão expostas as bases estruturais da democracia eletrônica para, finalmente, analisar as debilidades da figura. $\mathrm{O}$ anterior, com o fim de evidenciar que graças à tecnologia, a participação cidadã se vê facilitada e incentivada.

Palavras-chave: Internet, democracia eletrônica, sociedade da informação, participação política, tecnologias da informação e as comunicações (TIC), democracia direta, democracia representativa, participação cidadã. 


\title{
Internet como medio de promoción de la democracia*
}

\author{
Tatiana Monsalve López **
}

\section{SUMARIO}

Introducción - I. ASPECTOS PRELIMINARES: RESEÑA HISTÓRICA SOBRE DEMOCRACIA ELECTRÓNICA - II. SOPORTES ESTRUCTURALES DE LA DEMOCRACIA ELECTRÓNICA - A. Del ciudadano informado a la democracia de la transparencia - B. De un espacio público revitalizado a la democracia de debate - C. De la participación ciudadana a la democracia consultiva - III. LA FRAGILIDAD DE LA IDEA DE DEMOCRACIA ELECTRÓNICA - A. El ciudadano activo e informado, ¿mito o realidad? - B. El mito de la transparencia política - C. La democracia electrónica reducida al debate - D. Eliminación de intermediarios: ¿algo posible? - IV. CONCLUSIÓN: LA DEMOCRACIA ELECTRÓNICA, CAMINO POR RECORRER - Referencias.

Cómo citar este artículo: Monsalve López, T. (Junio, 2015). Internet como medio de promoción de la democracia. Revista de Derecho, Comunicaciones y Nuevas Tecnologías, 13. Universidad de los Andes (Colombia).

** Abogada y magíster en Derecho de la Universidad de los Andes. Representante legal de Asesores Jurídicos y Consultores Empresariales. Correo: tatiana_monsalve@yahoo.com 
Introducción

Las tecnologías de la información y la comunicación (TIC) intervienen cada vez más en nuestra cotidianidad. Como muestra de esto, Internet ha propugnado desde su nacimiento por la participación y la autonomía de sus usuarios. También se ha convertido en una herramienta innegable de deliberación y participación ciudadana, estableciendo nuevos modelos de información que permean todos los agentes dentro de la sociedad. En este sentido, cabe resaltar que la idea de las tic como instrumento de progreso democrático no es nueva.

Así, desde el siglo xIx se vio en el telégrafo un medio de asociación universal, que unía a Oriente con Occidente. Luego, la televisión fue imaginada como el conocimiento al alcance de todos y como un posible medio para ampliar el espacio público. Actualmente, Internet surge como una esperanza de renovación de nuestros sistemas políticos. Además, como una herramienta que bien utilizada puede ser valiosa para que los ciudadanos se conviertan en protagonistas e incidan en el mejoramiento de la sociedad, como alternativa al creciente malestar de la política, ${ }^{2}$ que encuentra sustento en la falta de legitimidad, desconfianza e inconformidad ciudadana en los actores políticos, reflejadas esencialmente en la apatía por esta actividad.

De esta manera, las redes electrónicas, al dar fluidez a la circulación de la información y faci-

2 Existe una progresiva necesidad de ampliar las zonas de participación ciudadana. Al respecto ver Cornwall y Coelho (2007). litar la interacción de los individuos, permiten a los ciudadanos participar más activamente en la vida pública. Por consiguiente, la tecnología conlleva el nacimiento de una democracia revitalizada: la electrónica. Este discurso proviene de múltiples fuentes, tales como: activistas, empresas comerciales, instituciones gubernamentales, académicos comprometidos, prensa y asociaciones. ${ }^{3}$

La noción de democracia electrónica no ha aparecido con la llegada de Internet; se ha desarrollado gradualmente desde la Segunda Guerra Mundial y la invención del computador. De este modo, podemos distinguir varias fases que dependen en primer lugar, del avance de la tecnología y en segundo lugar, de la evolución relacionada con la percepción de los problemas inherentes a la democracia.

En función de los contextos sociopolíticos y de las tic disponibles, la idea de democracia electrónica ha atravesado tres ciclos principales durante los últimos sesenta años. Primero, la etapa de la técnica vista desde la cibernética. ${ }^{4}$ Wiener es el principal exponente de este modelo encargado de estudiar la estructura de los sistemas por medio de la tecnología disponible, y cuya preocupación es: ¿Cómo la tecnología de la información puede contribuir al control racional de la sociedad y ser puesto al servicio de

3 Autores como Hagen, resaltan la existencia de variantes nacionales, que amplían las fuentes según el caso concreto. Ver Hagen, M. (1997).

4 Popularizada por el libro de Norbert Wiener: La Cibernética o el control y comunicación en el animal y la máquina, publicado en 1985. Con el fin de entender mejor las razones por las que se produce el fin del enfoque cibernético ver Meynaud (1964). 
una política más allá de la simple voluntad de los hombres? Posteriormente, surge la teledemocracia. ${ }^{5}$ Siguiendo a Etzioni, se materializa con el desarrollo de redes de televisión por cable, percibidas como medios de comunicación que podían ponerse al servicio de las comunidades, como una opción adicional al nacimiento de las redes locales. Por último, aparece el Internet como la nueva esperanza para la democracia, abriendo las fronteras de la política y especialmente de la participación ciudadana en el ciberespacio. En torno a esta última etapa girará el objeto central de este escrito.

En síntesis y gracias a esta evolución, el ciberespacio es percibido como un medio para salir de los sistemas políticos tradicionales, bajo una especie de sociedad virtual que a su vez absorbería el viejo mundo político real. Ya no se trata de aumentar la capacidad del Estado para interactuar con la sociedad, al igual que en la visión cibernética, o de revitalizar la relación social entre los ciudadanos como los defensores de la teledemocracia, sino de algo más radical: recrear la política.

Con este escrito pretendo demostrar que en el ámbito de la política, y específicamente en el de la democracia, el mejoramiento es apreciable gracias al uso de las nuevas tecnologías y más concretamente del Internet. Lo anterior, teniendo en cuenta que las herramientas tecnológicas

5 En teledemocracia, gracias al apoyo de la interactividad brindada por las redes de televisión por cable, se llevaron a cabo proyectos como Minerva, en Nueva Jersey, experimento que buscaba reconstituir electrónicamente las condiciones de participación democrática según el modelo griego. Ver Etzioni (1968). sirven para optimizar la comunicación y establecer proximidad entre representantes políticos y ciudadanos, otorgando, de este modo, poder a las personas al hacerlas más partícipes en los procesos de decisión política.

A continuación, con el fin de establecer la relación entre el uso de Internet y el aumento de estándares democráticos, realizaré (I) un estado del arte histórico sobre la evolución de la democracia electrónica. Luego, presentaré (II) los soportes estructurales y (III) las principales críticas a la democracia electrónica, para concluir porqué es fundamental ampliar los canales de participación ciudadana en el análisis de los estándares democráticos.

\section{ASPECTOS PRELIMINARES: RESEÑA HISTÓRICA SOBRE DEMOCRACIA ELECTRÓNICA}

A partir de los años 90 se abre una nueva etapa en la sedimentación de la idea de democracia electrónica, ligada, en primera medida, al desarrollo muy rápido -al menos en los países industrializados- de Internet. Debido a su organización anárquica, igualitaria, de cooperación, de la diversidad de formas de comunicación y de la libertad de expresión que autoriza, así como la relativa facilidad de su utilización, parece que Internet no solo ofrece nuevas soluciones a los problemas tradicionales de la crisis de la política. En este sentido, Internet constituye también un modelo de convivencia y se convierte en una metáfora para la nueva era de la política. Como ejemplo de participación ciudadana por este 
medio podemos citar enlaces virtuales como www.cause.com o www.thepetitionsite.com.

Esta inversión simbólica de la tecnología es alentada por el surgimiento de lo que se podría llamar la ideología californiana, representada por Barbrook y Cameron, ${ }^{6}$ inscrita deliberadamente dentro del movimiento de la globalización económica y opuesta a la intervención del Estado. Esta corriente consiste en el liberalismo económico y político alrededor de la figura de un ciudadano consumidor, promoviendo un individualismo hedonista y creativo que no excluye la solidaridad social o la conciencia ecologista de la fragilidad del medio ambiente y de deberes hacia las generaciones futuras. Norris Pippa, por ejemplo, desarrolla el tema de la cultura política de los internautas (2001).

Ahora bien, al hacer alusión al individualismo hedonista, comparto la posición de Lipovetsky (2000), en el sentido de afirmar que a pesar de partir de un concepto originalmente egoísta, la evolución de la sociedad ha hecho que se conjuguen el individualismo y la búsqueda del bienestar propio con los beneficios para la comunidad, reflejados en la sensibilidad ecologista, el culto de la participación, y la expresión y la valoración de lo local y de lo regional.

Basándose en esta doble mutación, la democracia electrónica ofrece una nueva concepción de la política que deja de girar alrededor del Estado-nación, para llegar a un ciberespacio abierto

6 Los principales representantes de la ideología californiana son Richard Barbrook y Andy Cameron. Disponible en http://www.imaginaryfutures. net/2007/04/17/the-californian-ideology-2/ sin territorio, sin jerarquía, reflexivo, en el que los individuos totalmente autónomos establecen relaciones múltiples.

El concepto del ciberespacio dio lugar a una profusión de visiones pudiéndose identificar dos corrientes principales: una, comunitaria, formulada por Howard Rheingold, que convierte a la comunidad virtual en la célula base de la nueva era de la política. Según este autor, gracias a las posibilidades interactivas de redes informáticas, Ios ciudadanos podrían adquirir fácilmente un capital social que les procuraría un poder real de acción política. ${ }^{7}$

Este tema de la comunidad virtual ha sido retomado por muchos propagandistas para acercarse a la concepción de Habermas (1993) del espacio público como lugar fundamental de la política. La política, según ese punto de vista, no se limita a participar, sino que conforma la sociedad, pensada como la asociación libre e igualitaria de individuos conscientes de su dependencia, unos respecto de otros, estableciendo entre sí lazos de mutuo reconocimiento. En términos de Delgado y Malet, el espacio público se convierte así en el "dominio en que ese principio de solidaridad comunicativa se escenifica, ámbito en que es posible y necesario un acuerdo interaccional y una conformación discursiva coproducida" (2011, pág. 59).

La otra corriente, más centrada en la economía política, y en este sentido más conservadora,

Teorización de la experiencia de red comunitaria Well (Whole Earth Electronic Link) de San Francisco, creada a finales de la década de 1980. Ver Rheingold (1994). 
está representada por la Progress and Freedom Foundation, a quien debemos la publicación en 1994 de la Magna Carta for the Knowledge Age, bosquejo de una teoría política del ciberespacio. ${ }^{8}$ Para ellos, la información se convierte en el principal recurso de las economías modernas, lo que altera las estructuras de la sociedad: el poder ya no se basa en bienes materiales, sino en la capacidad de comunicar. Puesta al alcance de todos por medio de Internet, la organización jerárquica de los sistemas políticos se cambia en provecho de formas de autoorganización, consensualmente inventadas por los ciudadanos.

Con el fin de contraponer las posiciones en torno a la noción de democracia electrónica procederé a establecer sus soportes estructurales y a destacar sus principales críticas. Esto último, teniendo en cuenta que la idea de democracia electrónica parece ofrecer algunas soluciones técnicas, pero trata parcialmente los problemas descubiertos, desde hace mucho tiempo, por la teoría de la democracia.

\section{SOPORTES ESTRUCTURALES DE LA DEMOCRACIA ELECTRÓNICA}

En los últimos años, una corriente de la literatura académica sobre la democracia electrónica se ha unido para caracterizar, en función de los modelos clásicos de la democracia, las formas que puede presentar en el uso de las tic y el Internet. Edwards (1995), por ejemplo, atraviesa

8 Disponible en http://www.pff.org/issues-pubs/futureinsights/ fi1.2magnacarta.html varias dimensiones de la democracia para distinguir tres versiones: una populista, una liberal y una republicana. Este mismo autor evolucionará en su reflexión, para encontrar finalmente seis tipos de democracia electrónica: ${ }^{9}$ directa, representativa, deliberativa, pluralista, asociativa y de consumo.

Hoff, Horrocks y Tops (2000), por su parte, extraen de conceptos tradicionales de ciudadanía cuatro modelos: consumista, elitista, neo-republicano y la ciberdemocracia, mientras que Van Dijk (2000), desde una perspectiva finalista, Ilega a seis modelos: legalista, competitivo, plebiscitario, pluralista, participativo y libertario. Por último, Schuler (1999) verifica si las prácticas políticas asociadas con Internet se encuentran en consonancia con los criterios conocidos de la democracia, propuestos por Dahl.

Este último enfoque es fructífero respecto de los interrogantes fundamentales que enfrenta cualquier organización política -la naturaleza del individuo, los alcances de la convivencia, la relación entre el individuo y la comunidad-y la variedad de arreglos propuestos por la fórmula democrática para responder a estas preguntas.

Asimismo, el análisis sobre la democracia electrónica resalta tres temas principales, en torno a los cuales se organiza: (A) la información de los ciudadanos, (B) el debate-discusión, (C) la

9 "I do this by distinguishing six models of democracy, each of which specifies a mechanism, appropriate institutions and intermediaries for achieving responsiveness. The six models are as follows: representative democracy, direct democracy, deliberative democracy, pluralist democracy, associative democracy and client democracy" (Edwards, 2006, pág. 5). 
deliberación y la toma de decisiones públicas. Cada uno de estos remite a problemas mayores de funcionamiento de los sistemas políticos existentes, a saber: la falta de transparencia en la política, la estrechez o el cierre del espacio público, la marginalidad de los ciudadanos en el proceso de toma de decisiones, como señalaré a continuación.

\section{A. Del ciudadano informado a la democracia de la transparencia}

Esta primera parte abarca la naturaleza de la información con la que pueden contar los ciudadanos. Se refiere al ideal de ciudadano informado, sostenido por filósofos liberales como Thomas Jefferson, quienes sustentan que para tomar buenas decisiones y participar plenamente en el proceso democrático se debe estar totalmente informado (Máiz, 2013).

En esta perspectiva, Internet $-\mathrm{y}$ especialmente la Web- es imaginada como una poderosa herramienta de información al servicio de la democracia. En comparación con los medios de información típicamente utilizados por los actores políticos (folletos, periódicos, etc.), Internet permite aumentar sin límite, a bajos costos y casi al instante la cantidad de información suministrada a los ciudadanos. También es capaz de mejorar la calidad de la información: reproducción de documentos originales, divulgación de documentos de gran tamaño, posibilidad de realizar investigaciones más precisas de manera rápida o vincular documentos almacenados en ubicaciones diferentes a través de motores de búsqueda. Por lo tanto, puede imaginarse to- dos los documentos relativos a un proyecto de interés público puestos a disposición, para consulta, de los habitantes de una ciudad.

Con base en lo anterior, la democracia electrónica se apoya en una de las carencias de las democracias liberales existentes: la libertad de comunicación, que se instaura como un principio fundamental, al que generalmente se otorga un valor constitucional dentro de los ordenamientos nacionales. Sin embargo, si la prensa goza de gran libertad, el derecho de los ciudadanos a la información -que les permite acceder a sus datos utilizados por los gobiernos o la administración-no se reconoce siempre. En este sentido, suele imponerse a las sociedades privadas la obligación de transparencia (datos sobre su capital, sus resultados financieros, publicaciones, etc.) de manera mucho más fuerte que a los gobiernos u organismos públicos.

En algunos casos, en los países donde existe un derecho de los ciudadanos a la información (por ejemplo, Estados Unidos con la Freedom of Information Act de 1964), este es aplicado arbitrariamente, se deniega el acceso a ciertos datos sensibles o se imponen varios obstáculos prácticos (fragilidad de los documentos originales, costos de reproducción elevados, falta de personal) que son invocados para restringir la comunicación de documentos (Catinat y Vedel, 2000). Las posibilidades técnicas actuales de digitalizar y poner en línea una gran cantidad de datos y la falta de transparencia de la acción pública ya no pueden justificarse por razones materiales y, por lo tanto, pueden resultar de una opacidad política o encubrimiento. 
En el caso colombiano, con la expedición de la Ley 1712 de 2014 se intenta llegar a la transparencia imponiendo a las entidades varios grados de confidencialidad, partiendo desde la información pública, pública clasificada, pública reservada y datos abiertos hasta la información privada por motivos de interés público, o reservada. En este último caso, el profesor Remolina establece:

La existencia de información que no debe ser conocida por el público (reservada) es una constante en todos los sistemas de gobierno. Quien posea este tipo de información tiene la obligación de abstenerse de publicar, comunicar o dejar consultar la misma a terceros o personas no autorizadas (2013, párr. 5).

De igual manera, Remolina señala, a partir de un estudio realizado por la OEA, que "la información es una herramienta esencial para combatir la corrupción, hacer realidad el principio de transparencia en la gestión pública y mejorar la calidad de nuestras democracias" (2013, pág. 312).

Sin duda, estas exigencias contribuyen a la concretización de principios relacionados con una mejor gobernanza o buen gobierno, que encuentra en Internet una de sus mejores y más eficaces herramientas.

\section{B. De un espacio público revitalizado a la democracia de debate}

El segundo soporte estructural de la idea de democracia electrónica se refiere a las formas y a la calidad del debate político. Internet es visto como un instrumento para estimular y enriquecer la discusión entre los ciudadanos. La información ya no representa un problema debido al aumento en el nivel general de conocimientos y a la existencia de muchos medios de comunicación. El tema central es la creación de un espacio público abierto, capaz de conciliar la expresión de múltiples ideas y aplicaciones que no pueden manifestarse en el marco institucional y rígido de la democracia representativa.

Específicamente, haciendo posibles las discusiones en foros de Internet, mensajería o listas de correo, los defensores de la democracia electrónica reconocen muchas virtudes susceptibles de revitalizar una verdadera democracia de debates, tales como:

- Es un lugar de libertad y autenticidad: cada persona puede expresarse sin trabas, en igualdad de condiciones al deshacerse de sus papeles institucionales o sus imágenes sociales (particularmente gracias al anonimato);

- Es un lugar que trasciende las fronteras geográficas, sociales y culturales: Internet pone en contacto a individuos de condiciones diversas, que sin la red no habrían tenido la posibilidad material de entrar en relación. Para un gran número de activistas de la democracia electrónica, Internet es sobre todo la herramienta que permitirá a una sociedad civil internacional crear contrapeso a la globalización de las sociedades y las estructuras de gobierno (Frederick, 1993);

- Es un lugar de entendimiento mutuo: la diversidad de sus usuarios hace de Internet un lugar 
en el que podemos ser confrontados con ideas y formas de pensar novedosas, donde se pueden compartir conocimientos, know-how; aún más, Internet permitiría a cada uno experimentar nuevos roles, logrando incluso romper sus propias limitaciones cognitivas;

- Es un lugar que genera vínculos sociales y condensa identidades colectivas: a través del intercambio, los participantes de los foros de discusión pueden tomar conciencia de sus problemas e intereses comunes, y por lo tanto de aquello que los une;

- Es un lugar de autorregulación: el Internet es visto como un espacio de debate en el que las reglas son inventadas y coproducidas - por el ajuste mutuo, por consenso- por todos los participantes.

Sin embargo, esta visión de democracia de debate a través de comunidades virtuales virtuosas, que Internet ha revelado, no es compartida con la misma intensidad por quienes rechazan la idea de democracia electrónica; en especial debido a las dificultades prácticas encontradas en los foros de discusión, a saber: mensajes de una minoría de participantes, predominio de la expresión de opiniones sobre el diálogo, comportamiento agresivo, entre otros.

\section{De la participación ciudadana a la democracia consultiva}

El tercer eje que organiza la idea de democracia electrónica se refiere a la participación de los ciudadanos en las decisiones políticas. Su campo de aplicación va desde una consulta ciudadana hasta la participación directa en el proceso de toma de decisiones. En concreto, se puede utilizar Internet para establecer una relación más estrecha entre funcionarios o elegidos y ciudadanos, aprovechando funciones como el correo electrónico (que facilita el contacto con funcionarios electos), los foros de noticias temáticas (donde cada ciudadano puede dar a conocer públicamente su opinión o sus propuestas), los cuestionarios en línea, o incluso el desarrollo de sistemas de votación electrónica. Respecto de este último punto, vale la pena consultar el mapa de voto electrónico con el fin de tener una percepción de los países en los cuales este se ha implantado o se pretende implantar. ${ }^{10}$

En el primer caso, la idea de democracia electrónica gira alrededor de la búsqueda institucional de una democracia más participativa o consultiva, a través de la implementación de dispositivos o procedimientos que involucran a más ciudadanos en el desarrollo de políticas públicas. Respecto de esta ingeniería institucional, el Internet puede ser concebido como un "simple" sistema de flujo de información que se suma a los mecanismos o dispositivos creados para la consulta de los ciudadanos o para hacerlos participar en la toma de decisiones públicas.

El Internet es esencialmente un facilitador técnico que simplifica y agiliza el contacto entre las personas, les permite ahorrar tiempo y aumentar sus intercambios. Pero también podemos pensar que el Internet es, en sí mismo, un vec-

10 Disponible en http://www.e-voting.cc/en/it-elections/world-map/ 
tor político adicional que genera una dinámica propia con efectos singulares. No obstante, siguiendo a Harto de Vera (2006), Internet no solo es un medio de comunicación conveniente entre gobernantes y gobernados, sino que puede crear un canal de expresión ciudadana que modifica la relación de poder entre gobernantes y gobernados, sin poner en entredicho el principio de la democracia representativa, al incrementar los canales y flujos de información para que los ciudadanos cuenten con una mayor fuerza en el proceso de toma de decisiones. ${ }^{11}$

Internet es una tic que ha impactado el mundo en prácticamente todos los escenarios: social, político, económico, cultural, etc. Para Castells (2001), Internet no solo es "una red de comunicación global” (pág. 21), sino un símbolo (pág. 306) de la nueva sociedad y "actualmente, las principales actividades económicas, sociales, políticas y culturales de todo el planeta se están estructurando por medio de internet" (pág. 17). Se podría hablar del mundo antes y después de Internet y de la "era digital y de la información" que ha cambiado, transformado y revolucionado ${ }^{12}$ varias cosas en nuestra sociedad. También se podría afirmar

11 "Como ejemplo de las proposiciones de este enfoque se sitúan las electronic town meetings. Estas asambleas fortalecen las relaciones entre representantes y representados y permiten a los representantes obtener un feedback de cuál es la voluntad de los electores a los que representan" (Harto de Vera, 2006, pág. 35),

12 "Internet está revolucionando las estructuras tradicionales del mercado al aportar una infraestructura común mundial para la prestación de una amplia gama de servicios de comunicaciones electrónicas. Los servicios de comunicaciones electrónicas disponibles al público a través de Internet introducen nuevas posibilidades para los usuarios, pero también nuevos riesgos para sus datos personales y su intimidad" (Considerando número 6 de la Directiva 2002/58/CE del Parlamento Europeo y del Consejo de la Unión Europea relativa al tratamiento de los datos personales y a la protección de la intimidad en el sector de las comunicaciones electrónicas (Directiva sobre la privacidad y las comunicaciones electrónicas)). que el mundo se divide entre personas conectadas y desconectadas a Internet.

Según Castells (2001):

Si la tecnología de información es el equivalente histórico de lo que supuso la electricidad en la era industrial, en nuestra era podríamos comparar el internet con la red eléctrica y el motor eléctrico, dada su capacidad para distribuir el poder de la información por todos los ámbitos de la actividad humana (pág. 15).

$Y$ agrega que "internet constituye actualmente la base tecnológica de la forma organizativa que caracteriza a la era de la información: la red" (Castells, 2001, pág. 15).

Así, la idea de democracia electrónica puede, de manera más radical, ser presentada como medio para lograr una democracia directa. Esta visión, impregnada en gran parte por la concepción de Rousseau sobre la política (Marks, 2001), establece que la soberanía no puede alienarse y no puede haber decisión soberana sin la opinión de cada uno sobre el tema; se basa en un razonamiento en tres etapas:

1. Hay una forma óptima de democracia, representada por el ágora ateniense, que pudo ser practicada mientras se mantuvo reducida al espacio político de la ciudad;

2. La democracia representativa es un recurso que no pudo imponerse cuando los sistemas políticos se extendieron en vastos territorios que contaban con millones de personas, debido a la imposibilidad física para reunir a todos los ciudadanos. 
3. El Internet, al permitir técnicamente consultar millones de personas y casi instantáneamente obtener su voto en una decisión, hace factible una democracia directa a gran escala.

No obstante, independientemente de verificar si el Internet es viable técnicamente para soportar votos electrónicos masivos, podría decirse que este enfoque hace una lectura un tanto precipitada de los teóricos de la democracia representativa. Para ellos, la democracia representativa posee una concepción de la política en la que los ciudadanos no son igualmente aptos para deliberar sobre los asuntos públicos y la elección no es una transferencia de voluntad, sino una forma de selección de los ciudadanos más capaces.

En este sentido, y haciendo referencia al empoderamiento del individuo, Internet también es una herramienta que confiere poder a las personas. Como ejemplo de ello encontramos páginas en la red como www.change.org, que demuestra cómo cualquier persona puede lograr cosas importantes con Internet. Para complementar esta idea, cabe mencionar al profesor Cano, quien señala:

Hace más de 10 años Internet era un sitio donde algunos generaban los contenidos y decidían qué se mantenía allí o no. Hoy, luego de una impresionante evolución de la Web 2.0, somos las personas las que desarrollamos y actualizamos los contenidos que se advierten en Internet (2013, pág. 136) .

Según Cano (2013), “pasamos de un control de pocos a una participación de muchos" (pag.
136). Pero no solo eso, sino que las tic e Internet han facilitado que cualquier persona tenga poder informático porque está en capacidad de recolectar información sobre otros y utilizarla con múltiples propósitos. Este poder en cabeza de las personas fue reconocido en noviembre de 2013 por la onu, con ocasión de la expedición de la Resolución sobre "El derecho a la privacidad en la era digital”. Esta organización destacó que:

El rápido ritmo del desarrollo tecnológico permite a las personas de todo el mundo utilizar las nuevas tecnologías de la información y las comunicaciones $\mathrm{y}$, al mismo tiempo, incrementa la capacidad de los gobiernos, las empresas y las personas de llevar a cabo actividades de vigilancia, interceptación y recopilación de datos (onu, 2013, pág. 1).

\section{LA FRAGILIDAD DE LA IDEA DE DEMOCRACIA ELECTRÓNICA}

La democracia electrónica se evalúa generalmente con base en sus consecuencias sobre los sistemas políticos, que pueden ser tanto positivas (una mayor participación en la vida política, revitalización del espacio público) como negativas (la creciente desigualdad entre los ciudadanos, control social); sin embargo, difícilmente se examina desde el punto de vista de sus posibilidades. Esto implica una crítica interna de la idea de democracia electrónica: ¿Los supuestos en que se basa son válidos o realistas?, ¿trata sistemáticamente los principales problemas que implica toda organización política y que los teóricos de la democracia han planteado desde 
hace tiempo?,13 ¿es consistente con el análisis de los medios propuestos? Al respecto, y con el fin de exaltar los principales problemas que ha tenido que enfrentar la democracia electrónica, se plantean los cuatro puntos o interrogantes siguientes:

\section{A. El ciudadano activo e informado, ¿mito o realidad?}

El discurso sobre la democracia electrónica se basa en un concepto muy exigente de la ciudadanía. En la era del hipertexto, el ciudadano "bueno" sería un ciudadano hiperactivo que se informa permanentemente y compara múltiples fuentes, que participa regularmente en las discusiones y consultas y que controla constantemente a los elegidos. ${ }^{14}$ La idea de democracia electrónica reconoce un supuesto implícito: la mayoría de los ciudadanos están dispuestos a participar intensamente en la vida política y esta participación parte de una mejor información. Esta visión de la ciudadanía, que nos reenvía a los teóricos de la democracia del siglo XIX (Mill, Locke o Tocqueville), sobrestima, sin duda, la apetencia de los individuos por la política.

Por otro lado, las investigaciones emanadas de la socio-psicología cognitiva enfatizan que los ciudadanos son animales políticos perezosos que se esfuerzan por economizar su energía: gran parte de su actividad consiste en no bus-

13 En este sentido convendría ampliar el concepto de Castells (2001), según el cual si bien Internet no puede entrar a solucionar tecnológicamente los problemas de la democracia, sí aporta, de manera indudable, una nueva dinámica a la política.

14 Al respecto, ver Dahl (1993). car más información sino implementar procesos para filtrar, reducir y gestionar la información superabundante que reciben (Popkin y Dimock, 1999).

Los propagandistas y activistas de la democracia electrónica se enfrentan a la compleja relación entre ciudadanía e información. Si en cualquier democracia la información es una necesidad, se hace necesario ser capaz de ordenar y evaluar la información que se recibe. Este proceso implica establecer límites de referencia y de juicio.

Así, el Internet por sí solo no resuelve este problema, sino que hace más compleja la tarea: el exceso de información puede causar inhibición del ciudadano o ponerlo en la imposibilidad de decidir. Con autores como Norris, puede abrirse otra perspectiva: ¿En lugar de deplorar la insuficiente información de los ciudadanos y su conocimiento imperfecto de los asuntos públicos (falacia de educación cívica), en vez de aceptar una visión limitada de la ciudadanía donde los electores se conforman con representaciones políticas aproximadas (la falacia relativista), no convendría más interesarse en el nivel de conocimientos prácticos necesarios para ser un ciudadano pleno?

Además, siempre que sea razonable pensar que todos los ciudadanos no desean participar activamente en la vida política, ¿cómo conciliar la apatía de algunos y el activismo de otros?, ¿cómo fomentar en los ciudadanos más activos actitudes de sumisión y esta cultura cívica mixta que, según Almond y Verba (1989), son esencia- 
les al funcionamiento de la democracia? Para los teóricos de la democracia representativa esta tensión estructural se resuelve mediante las elecciones generales periódicas, concebidas como momento de encuentro entre ciudadanos activos y pasivos, y como un medio de superar el conflicto e instalar, durante un determinado período, un orden social que permite al "gobierno gobernar".

Con todo, la idea de democracia electrónica no proporciona ninguna respuesta a este problema. Postula un estado de permanente politización que, por un lado, es capaz de excluir permanentemente a los ciudadanos menos activos y, por otro, expone de manera frecuente a los ciudadanos más activos a situaciones de decepción, multiplicando las posibilidades de conflicto.

\section{B. El mito de la transparencia política}

El requisito de transparencia de la acción pública es inherente a la democracia. Representa a la vez la condición, el medio y el fin. Primero, permite verificar el cumplimiento de los principios de la democracia y de las reglas constitutivas del contrato social, comenzando por la igualdad de los individuos ante la ley. Representa un derecho de control de la sociedad civil sobre el sistema político al permitir que cada ciudadano pueda emitir un juicio sobre la labor de los representantes elegidos.

También puede pensarse que la transparencia mejora la calidad del proceso de toma de decisiones: promueve la movilización de grupos cuyos intereses pueden estar en riesgo, ayuda a construir la crítica, obliga a la administración a afinar sus argumentos o a modificar sus pautas. ${ }^{15}$ Por el contrario, el oscurantismo tiende a proteger un poder arbitrario sin obediencia de reglas o a ocultar la acción de los grupos que persiguen sus propios intereses. ${ }^{16}$ La democracia electrónica pretende contribuir con una mayor transparencia a la acción del Gobierno, proporcionando una herramienta potente y de bajo costo, de almacenamiento y distribución de la información, convirtiendo en obsoletos los argumentos materiales que utilizan los Gobiernos para impedir el acceso a los documentos públicos.

Sin embargo, el problema de la transparencia no está completamente resuelto. En primer lugar, el Internet no elimina las barreras -cognitivas y sociales- que hacen que algunos grupos sean más capaces que otros para acceder a los datos que sustentan la acción pública. Estos últimos cuentan no solo con mayores recursos financieros, sino también con una mayor capacidad para identificar, localizar y procesar la información relevante basada en enlaces establecidos con los diferentes sectores del aparato administrativo.

En este sentido, Internet podría resultar, intencionalmente o no, convertido en un instrumento de opacidad: aumentando la cantidad de datos facilitados a los ciudadanos, se perjudica a aquellos menos preparados para manejar un

15 Sobre la importancia de la transparencia en política, ver Moreno Jiménez (2006).

16 Ver Neveu (1997) 
exceso de información. La verdadera transparencia de la acción administrativa, en segundo lugar, implica un nuevo acondicionamiento de las relaciones del Estado con la sociedad civil. La opacidad que la administración mantiene tan a menudo, según Vedel (1994) viene de su subordinación a la autoridad política (jurídicamente, la administración no está obligada a rendir cuentas directamente a los ciudadanos) y a los principios de generalidad y de igualdad que organizan su funcionamiento (la administración no puede proporcionar respuestas personalizadas a las preguntas planteadas por los ciudadanos).

Para actuar mejor en la sociedad, el Estado se fragmenta en unidades especializadas, multiplica los niveles de intervención, establece relaciones con un número creciente de socios, tecnifica sus operaciones. En otras palabras, para servir mejor a los ciudadanos, el Estado se convierte en una máquina cada vez más compleja y menos comprensible para el público. La búsqueda de un funcionamiento más democrático de la acción pública puede, incluso, caer en contradicción con la exigencia -también democrática- de transparencia por cuanto se traduce en una profusión de normas de procedimiento, manejadas solo por especialistas. Así, conviene cuestionarse si el deseo de más transparencia no proviene de una visión ingenua de la acción política en la cual se presume la total honestidad de las partes.

\section{La democracia electrónica reducida al debate}

El discurso de la democracia electrónica tiende a reconocer una gran importancia al debate y a la discusión, al punto de dar la impresión que se reduce a la posibilidad de un escenario público. Maravillados por la cantidad de diálogos que se forman por Internet, algunos activistas de la democracia electrónica están adoptando una visión rudimentaria de lo que es, o debería ser, un debate en una democracia, descuidando casi por completo su dimensión política.

Otra debilidad del discurso sobre la democracia electrónica, respecto del tema del debate, es la poca atención que se concede a su carácter democrático. Además de la afirmación del principio de igualdad entre los participantes hay poca reflexión sobre los fenómenos de dominación, descalificación y manipulación que pueden existir en una discusión. Planteada como principio de la democracia electrónica, la libertad de expresión cuenta con una definición muy amplia, por lo que en un gran número de casos el debate político podría reducirse a una lluvia de ideas de los participantes.

En este sentido, los partidarios de la democracia electrónica raramente se cuestionan sobre las limitaciones o los efectos derivados del carácter escrito de los foros de discusión, aunque sabemos que una proporción significativa de la población cuenta con dificultades de comunicación escrita. Finalmente, al centrarse en la discusión pública, el discurso sobre la democracia electrónica todavía dice poco acerca de 
los medios y procedimientos que permiten a los ciudadanos ir más allá del intercambio y la confrontación de sus opiniones para la toma de decisiones.

\section{Eliminación de intermediarios: ¿Algo posible?}

Por otra parte, el discurso de la democracia electrónica tiende a considerar que los órganos intermediarios -como los partidos políticos, los sindicatos y los medios de comunicación- pervierten el funcionamiento de los sistemas políticos. Ubicados entre los gobernantes y los gobernados, contaminarían la comunicación entre ellos y amoldarían, según intereses propios, la expresión de los ciudadanos. El discurso sobre la democracia electrónica se une a una crítica antigua y a veces populista de la democracia representativa, nutrida por la disputa más contemporánea del papel de los sindicatos y los medios de comunicación. Los primeros son asimilados a órganos de gestión integrados en las estructuras neocorporativas del Gobierno, más para asegurar una mayor estabilidad del sistema político que para la defensa de los trabajadores. Los últimos son acusados de disfrazar la realidad, de excluir la representación de opiniones discrepantes y eventualmente de participar en la consolidación del orden social.

Por el contrario, Internet debe evitar estos mediadores promoviendo una libre coordinación de ciudadanos y una búsqueda de solución a sus quejas, al ofrecer un sistema alternativo de información sin "filtros", que pondría fin a la pasividad de los receptores.
Sin embargo, al criticar a los cuerpos intermediarios el discurso sobre la democracia electrónica crea un conflicto respecto de varias cuestiones relacionadas con las funciones de mediación. En primer lugar, se encuentra la adhesión de los intereses particulares a las reivindicaciones colectivas y a sus prioridades en los programas de acción.

En segundo lugar, las instancias de mediación proporcionan marcos de referencia y de interpretación que reducen el exceso de información que los ciudadanos tienen que administrar. Si bien los medios de comunicación pueden distorsionar la realidad, también realizan un trabajo de simplificación y traducción que facilita la decodificación de la información.

Asimismo, los partidos políticos proporcionan lecturas del mundo. Además, como obsesionado con la inmediatez de las herramientas de comunicación, el discurso de la democracia electrónica no toma en cuenta la inclusión en el tiempo de la vida política ni aborda el tema de la socialización o la memoria política. Se tiene la impresión de que los ciudadanos de la era electrónica entran en contacto con el sistema político, con una cultura y un almacenamiento de experiencias y conocimientos ya existentes, sin que se sepa de qué manera se han constituido y cómo se asegura su transmisión.

No obstante, la observación de los usos de Internet requiere que los defensores de la democracia se cuestionen acerca de la mediación, dado que la red revela nuevos mediadores (motores de búsqueda, portales de información, provee- 
dores de información). Además, los sujetos más activos, aun en Internet, suelen ser los individuos mejor integrados en el proceso político (Kenneth, Berry y Goldman, 1989).

Cabe preguntarse si algunos de ellos, aprovechando sus habilidades sociales, no están adquiriendo gradualmente un papel central en la producción y organización de la información electrónica, que defiende sus propios objetivos y la autopromoción de sus miembros activos.

\section{CONCLUSIÓN: LA DEMOCRACIA ELECTRÓNICA, CAMINO POR RECORRER}

Luego de haber plasmado los diferentes debates teóricos y dogmáticos alrededor del problema planteado dentro del presente escrito, así como las diversas dificultades que pueden surgir respecto de la aplicación práctica de la tecnología dentro de la democracia, se evidencia que con la utilización de las tıc se busca un ideal.

En este sentido, Cécile Beatrix (2000) señala que "el énfasis se hace sobre el efecto educativo de la participación ciudadana y su impacto positivo en el desarrollo del individuo dentro de la sociedad" (pág. 88). Sin embargo, en términos de Thierry Vedel (1994), a menudo se suele asumir la existencia de un ciudadano modelo, hiperactivo, sin tener en cuenta las dificultades que muchas personas enfrentan.

De acuerdo con lo anterior, cabe mencionar que las inequidades pueden abarcar temas tan bá- sicos como la disponibilidad o el tiempo que se tiene para participar activamente en la sociedad. Respecto de este tema, Jean-Pierre Durand (1997) afirma que "las fuerzas sociales susceptibles de transformarse en movimiento social necesitan no solo medios económicos, sociales y culturales sino también deben contar con el factor tiempo" (pág. 157).

Por otra parte, conviene mencionar que a pesar del poder ilimitado que parecen tener las TIC y más concretamente Internet, este solo podrían alcanzar una verdadera influencia en la política en la medida en que la base social se lo permita. Lo anterior, teniendo en cuenta que un gran número de problemas dentro de las democracias actuales provienen de comportamientos culturales más que de opciones tecnológicas puestas a disposición. Únicamente por medio de la toma de conciencia de las personas dentro del contexto político y del establecimiento de un sentido de pertenencia podrían aprovecharse al máximo las potencialidades que ofrecen estas herramientas a la política. Sin embargo, no puede negarse que el Internet contribuye a una política más transparente, gracias a mecanismos novedosos de participación que permiten, por medio de la información, crear ciudadanos capaces de controlar la clase política.

Paralelamente a la inquietud anterior, ¿cómo lograrían Internet y la tecnología garantizar una mayor intervención ciudadana, motivando a participar no solamente a aquellos que lo han hecho siempre, sino transformando a la democracia en algo más atractivo para todos? 
Las TIC actualmente son utilizadas no solo para entregar servicios a la población, sino para interactuar con la administración en virtud del nuevo poder ciudadano. Sin embargo, la contribución de las TIC en temas de participación no puede limitarse a reforzar el proceso democrático por medio de la información. Deben buscarse nuevas pautas basadas en el uso de las tecnologías disponibles, aunque esto no parezca viable teniendo en cuenta que hasta ahora el poder de los gobiernos se ha basado en gran medida en el control de los flujos de información.

Por lo antes mencionado y a pesar de los riesgos que implique intentarlo, me parece que las posibilidades ofrecidas por la tecnología merecen la pena ser aprovechadas, porque cada vez la sociedad se "virtualiza" más y porque las generaciones futuras contarán con un mayor apego a todas estas estrategias y opciones en la red.

\section{Referencias}

Almond, G. y Verba, S. (1989). The Civic Culture. Political Attitudes and Democracy in Five Nations. Newbury Park, California: Sage Publications, Inc.

Barbrook, R. y Cameron, A. (2007). The Californian Ideology by Richard Barbrook and Andy Cameron. Recuperado el 10 de mayo de 2014 de Imaginary Futures: http://www.imaginaryfutures.net/2007/04/17/the-californian-ideology-2/

Béatrix, C. (2000). La "démocratie participative" de mai 68 aux mobilisations anti-TGV. Proces- sus de consolidation d'institutions sociales émergentes, (Thèse de doctorat en science politique). Paris: Université de Paris I Sorbonne.

Cano Martínez, J. J. (2013). Inseguridad de la información: una visión estratégica. Bogotá: Alfaomega.

Castells, M. (2001). La galaxia internet: reflexiones sobre internet, empresa y sociedad. Barcelona: Plaza \& Janés.

Catinat, M. y Vedel, T. (2000). Public policies for digital democracy. En J. Van Dijk y K. Hacker, Digital Democracy: Issues of and Theory of Practice (págs. 184-208). London: Sage Publications Ltd.

Cornwall, A. y Coelho, V. S. (Eds.). (2007). Spaces for change?: the politics of citizen participation in new democratic arenas. London: Zed Books.

Dahl, R. A. (1993). La democracia y sus críticos (Vol. 779). Barcelona: Paidós.

Delgado, M. y Malet, D. (2011). El espacio publico como ideología. Madrid: Los Libros de la Catarata.

Durand, J. P. (1997). La pensée critique peutelle être utile? La Revue du M.A.U.S.S., (9), págs.151-167.

Edwards, A. (1995). Informatization and views of democracy. En W. BHJ van de Donk, I. Th. M. Snellen y P. W. Tops (Edits.), Orwell in Athens. 
A perspective on informatization and democracy (págs. 33-49). Amsterdam: IOS Press.

Edwards, A. (2006). ICT strategies of democratic intermediaries: A view on the political system in the digital age. Information Polity, (11), 163-176.

Etzioni, A. (1968). The Active Society: a Theory of Societal and Political Process. New York: Free Press.

Frederick, H. (1993). Computer Networks and the Emergence of Global Civil Society. En L. Harasim (Edit.), Global networks: Computers and international communication (págs. 283295). Cambridge, Massachusetts: MIT Press.

Habermas, J. (1993). The Structural Transformation of the Public Sphere. An Inquiry into a Category of Bourgeois Society. Cambridge, Massachusetts: MIT Press.

Hagen, M. (Marzo de 1997). A Typopogy of Electronic Democracy. En Martin Hagen [en línea]. Consultado 22 de abril de 2014. Recuperado de: http://martin-hagen.net/publikationen/ elektronische-demokratie/typology-of-electronic-democracy/

Harto de Vera, F. (2006). Tipologías y modelos de democracia electrónica. Revista de Internet, Derecho y Política, (2), 32-44.

Hoff, J., Horrocks, I. y Tops, P. (2000). Technology and social change: the path between technological determinism, social constructivism and new institutionalism. En I. Horrocks, J.
Hoff y P. Tops (Edits.), Democratic Governance and New Technology: Technology Mediated Innovations in Political Practice in Western Europe, (págs. 13-33). London: Routledge, 2000.

Kenneth, J., Berry J. y Goldman, J. (1989). The Challenge of Democracy: Government in America. Boston: Houghton Mifflin Company.

Lipovetsky, G. (2000). La era del vacío. Barcelona: Anagrama.

Máiz, R. (Octubre-Diciembre de 2013). Naturaleza, Nación y República Federal: el excepcionalismo norteamericano de Thomas Jefferson. Revista de Estudios Políticos (nueva época), (162), 13-38.

Marks, J. (Summer 2001). Jean-Jacques Rousseau, Michael Sandel and the Politics of Transparency. Polity, 33(4), págs. 619-642. DOI: $10.2307 / 3235519$. Obtenido de jstor home <http://www.jstor.org.ezproxy.uniandes.edu.co:8080/stable/3235519>

Meynaud, J. (1964). La technocratie. Mythe ou réalité? Paris: Payot.

Moreno Jiménez, J. M. (2006). E-cognogracia: nueva sociedad, nueva democracia. Estudios de Economía Aplicada, 24(1), 313-333.

Neveu, E. (1997). Une société de comunication? París: Montchrestien.

Norris, P. (2001). Digital Divide. Civic Engagement, Information Poverty, and the Internet 
Worldwide. Recuperado el 28 de abril de 2014 de jstor: http://www.jstor.org.ezproxy.uniandes.edu.co:8080/stable/10.1086/378471

Organización de Naciones Unidas. Asamblea General. (20 de noviembre de 2013). El derecho a la privacidad en la era digital. Sexagésimo octavo periodo de sesiones. A/C.3/68/L.45/ Rev.1.

Popkin, S. y Dimock, M. (1999). Political Knowledge and Citizen Competence. En S. Elkin y K. Soltan (Edits.), Citizen Competence and Democratic Institutions (págs.117-146). Pennsylvania: The Pennsylvania State University Press.

Remolina Angarita, N. (2013). Tratamiento de datos personales: aproximación internacional y comentarios a la Ley 1581 de 2012. Bogotá: Legis.

Remolina Angarita, N. (Marzo 26 de 2014). Impacto de la nueva ley de acceso a la información en el tratamiento de datos personales. Ambito Jurídico, (24).
Rheingold, H. (1994). Realidad virtual: Ios mundos artificiales generados por ordenador que modificarán nuestras vidas (E. Fisher, Trad.). Barcelona: Editorial Gedisa.

Schuler, D. (1999). How Do We Institutionalize Democracy in the Electronic Age?. Communication \& Strategies, (31), 79-91.

Van Dijk, J. (2000). Models of Democracy and Concepts of Communication. En J. van Dijk y K. Hacker (Edits.), Digital Democracy. Issues of Theory and Practice (págs. 30-53). London: Sage Publications Ltd.

Vedel, T. (1994). Introduction à une socio-politique des usages. En A. Vitalis, Médias et nouvelles technologies de communication. Pour une socio-politique des usages (págs. 13-35). Rennes: Editions Apogée.

Wiener, N. (1985). Cibernética o el control y comunicación en animales y máquinas. Barcelona: Tusquets. 